\title{
Moodgroove - Creative expression to your mood
}

\author{
Suraj Hattangadi \\ FIRSTusable \& UXPlacebo.com \\ 124, MM Road, Bangalore \\ suraj@firstusable.com
}

\author{
Mahalakshmi Viswanathan \\ UXPlacebo.com \\ 124, MM Road, Bangalore \\ mahalakshmi@firstusable.com
}

\author{
Suyog Deshpande \\ FIRSTusable \\ 124, MM Road, Bangalore \\ suyog@firstusable.com
}

\begin{abstract}
Mood tracking in the new realm of web 2.0 applications has undergone many interpretations and visualizations. On one extreme are the fun social applications which greatly trivialize mood tracking to a scale of emoticons while on the other hand there are those which complicate the idea by making the application highly technology oriented and bordering on scientific and statistical analysis.
\end{abstract}

\begin{abstract}
To strike the right balance between fun and seriousness, we worked on finding a solution for a single question - what would each one of us, as a common user, conversant with the social media paradigm, want in a mood tracker that proves to become a mood diary as well as a social networking medium? Moodgroove maintains the spirit of self-tracking mood (for introspection and understanding), while allowing synergic fusion of self-realization value as well as social expression. It satiates not only the stated but also the perceived needs of users. Moodgroove enables users to locate their mood on a scale, express it in words and associate reasons to it. The product visualizes these factors across time, and shows the patterns in users mood variations. What brings thrill to this product is its ability to convert a mood entry into a piece of art - a traditional medium to express mood. Moodgroove translates the mood into abstract art with colors and interaction patterns, while creating a musical mood piece for the specific mood. Yet another feature is the generation of a short poem based on the user's selection and choice of words to express his mood. There are potential possibilities and options to selectively share Moodart; Moodtune and Moodmuse on Facebook or Twitter bringing social aspect to the product.
\end{abstract}

In this case study, we delineate the various challenges faced, experimental techniques developed and the evolution of Moodgroove from what it started out to be - an interaction that would encourage people introspect and realize how their moods are influenced by various aspects in their lives

Mood Tracking, Social Media, Mood Expression, World Mood Temperature, Experiential Sampling Method.

\section{INTRODUCTION}

As economies fail and layoffs become the way of the world, more and more people start thinking about "existentialism" and coping with their realities. In such a situation, applications in the domain of "Introspection Space" start gaining attention. Capturing and analyzing emotions and mood is considered to be an important factor for introspection.

Moodgroove has been a journey which started with a simple idea of mood tracking and finally culminated into an application that not only captures mood but also presents it in various manifestations. As the topic of mood capturing is sensitive, throughout our journey we encountered several challenges pertaining to human emotion and interface design. In this case study, we elaborate on how these challenges led to the evolution of Moodgroove concept and opened up a plethora of possibilities in the mood space.

\section{THE MOOD SPACE}

Although moods and feelings have the potential to take social networking to the next level, the focus of current Web 2.0 applications have primarily been to keep it simple and fun - with the use of emoticons. However, by keeping it simple and fun, most of these applications trivialize the depth and complexity of human emotions and feelings. One cannot truly express his/her mood because of the 
restricting vocabulary provided by these applications.

On the other extreme, there are also applications [1] which are specifically built for tracking moodannotated data from blogs and online posts and represent this data in the form of graphs. They thrive on the existing content on the web, and act as world mood aggregators. These graphs, however, are complex and may neither be interesting, nor easy to understand, to a common user. With the above mentioned studies, we defined our problem statement as - what would each one of us, conversant with the social media paradigm, want in a mood tracker that evolves into a mood diary as well as a social networking medium?

\section{INSPIRATIONS}

While surveying the mood space, we also happened to chance upon a few applications which truly stand out with respect to their treatment of mood data- either in the form of visualization (e.g. We Feel Fine) or in the exploration of mood with another medium (e.g. Gettyimages- Moodstream)

\subsection{We Feel Fine}

We feel fine is an experimental project started by Jonathan Harris that captures the world's expression by collating every bit of feeling and emotion mentioned in blogs across the web. More than just capturing this information, it's the way this information is visualized that makes it very inspiring. The same information is expressed as various movements - Madness, Murmurs, Mobs, Montage, Metrics and Mounds. Each of these movements, represent mood information in different forms. [2] For example the screenshot below shows the "Madness" movement - each dot in the space represents a feeling captured from a recent blog entry. The dots are colour coded to indicate the approximate feeling -happy positive feelings are indicated by bright colours and sad negative feelings by dark colours. Clicking on any dot brings up the sentence that captures the feeling from the corresponding blog entry.

\subsection{Gettyimages- Moodstream}

This application explores the interplay between mood and visual/video footage. Based on a group of settings, the application generates a stream of visual and video footages from Getty repository. It is a visual brainstorming tool specifically designed to take you in inspiring, unexpected directions. As you watch and listen you are exposed to different images which can stimulate your mind and help you think of new concepts. You can create a series of "moodboards" that consist of a combination of soundtracks, Getty images \& videos - based on sliders describing tagged asset attributes, such as happy vs. sad, warm vs. cool, or nostalgic vs. contemporary. It also inspires the idea of having users create their own Moodstream - including their own videos, favourite tracks and pictures.

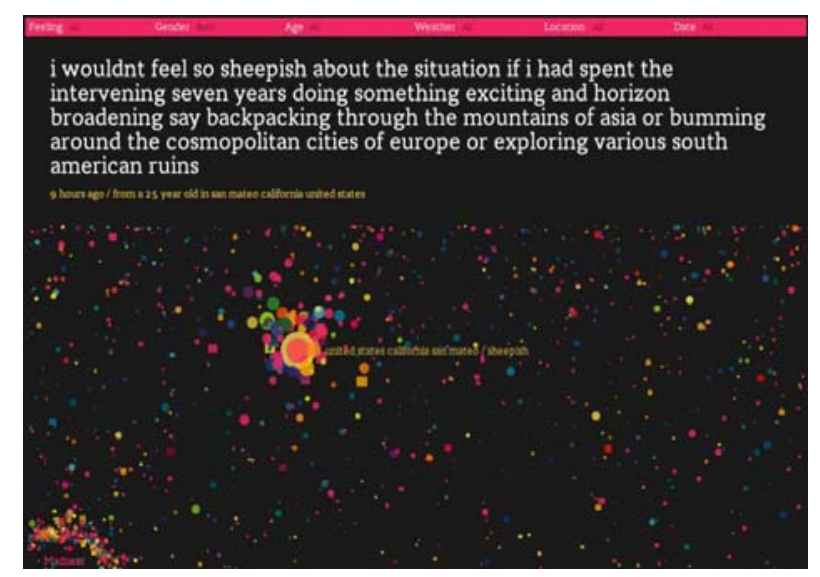

Figure 1: Screenshot of We feel fine.

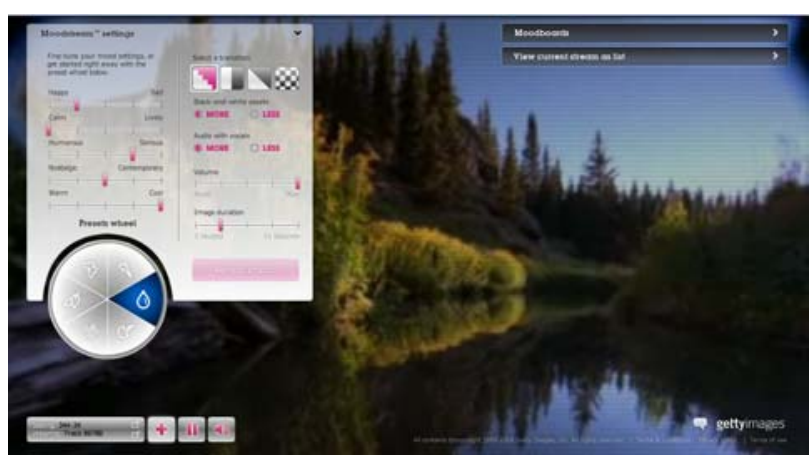

Figure 2: Screenshot of Gettyimages-Moodstream.

\section{CONCEPTUALIZATION}

We have discussed and involved many psychiatrists, psychologists and neurosurgeons during the brainstorming sessions of this product on a non-commercial basis and solely for the validation of certain aspects of Moodgroove.

These sessions helped us in crystallizing what we wanted our product to be, and the major issues it had address - keeping the system simple but not trivial, building in flexibility, and bringing value to each and every user in the short and long term.

On another plane, an important factor to be considered was whether the system should be leading or non-leading. By leading, we mean, should the system suggest feelings which could be picked by the user - which means there is a certain amount of influence that the system exercises on the user simply by displaying a set of words which knowingly or unknowingly the user ends up picking, 
even though he may not quite feel that way. On the other hand, building an honest system, meant keeping it completely open ended - asking a question like "How do you really feel" which makes the user think and come up with his own word/phrase from his vocabulary (this is clearly a clinical approach favoured by psychiatrists because of its unbiased nature). However, research shows [3] that the latter approach may not work very well with users simply because most people find it easier to pick from the choices offered rather than having to come up with their own. It also, in a way, ensured that users might not end up using the same kind of trivial words like "I feel - fine" which is what we all are pre-programmed to say and think, no matter how we really feel deep down.

Having chosen to go the "leading" route, the next step was to design the steps involved in mood input. To keep it simple, we started off with a familiar component - the slider with grooves ranging from most positive mood right up to most negative mood, so users could indicate generally which mood-groove they were at before drilling down to their feelings.In order to build in flexibility, we felt the need to go beyond just having the groove scale, and so we introduced what people most often use to express their feelings - a huge cloud of "feeling" words. Not only were there hundreds of words to pick from, you could also pick any word against any groove value, so there were no hard limitations on word-groove pairing.

While in the midst of designing the mood-input process, we came across a technique in psychology called Experiential Sampling Method[4]. This method provides guidelines for users to keep track of their feelings over a period of time, along with a record of why they felt the way they did corresponding to each mood entry. This method appealed to us and helped us resolve the third thing to be addressed - the value of this product if we could build a system which not only kept track of your feelings over a period of time, but also maintained a record of why you felt that way, it could provide immense value for users - they could find patterns in their moods and the reasons linked to them.

Once the core interaction was figured out, what remained was the visualization of this recorded mood data. Here we intended to demonstrate true value to the user by showing not just his mood variation as a plot across time, but also patterns in his mood variations and their associated reasons. This could stimulate introspection and selfrealization, if a user used Moodgroove on a regular basis.

Another area we were really keen on exploring was the expression of mood in various forms - art, music and literature. Our literature search [5] revealed the following findings.

Art: It is well known that art, and specifically color influences our mood and our thoughts. Each color has a psychological value and it affects people depending upon one's age, gender, climate and ethnic background. It is found that red raises the whole energy level, blue brings down blood pressure, slows respiration \& heart rate and Orange evokes excitement. Instead of exploring the influence of colour on our moods, we experimented with the reverse relationship - expressing mood through art.

Music: Known to be the international language, has great effect on our mood. Certain tunes and beats tend to get a similar reaction from most people. Indian classical music is known to be energizing, soft lullaby-like music to unwind, music with specific pace of beats evokes enthusiasm, medium-fast to fast music is good during exercise and housecleaning. Just as music could influence mood, there is scope for expressing mood in terms of music. This was another form of expression that we were keen on exploring and coming up with a tune generator which could establish this connection.

Literature: Every piece in literature has a certain character and mood associated with it, which reflects the author's personality or that of the characters in it. Therefore, achieving mood in literature, especially in poetry has been an age-old tradition. Just that there was always a person behind each poem, while here we intended Moodgroove to be the creator, and the poem to be a reflection of the user's mood.

\section{EVOLUTION}

\subsection{Moodgroove - where it all started}

The idea started a year ago when we decided to create a product that would allow people to gauge their mood temperature. This was measured on a 9-pt scale, which had one word associated with each scale value. It was however, a very limiting system because it only allowed for people to indicate their mood across a 9-word expanse.

This model also had a very basic Report in the form of a Calendar View for day, week, month and longer periods and a Chart view which could plot the moods across a period of time as a graph. 


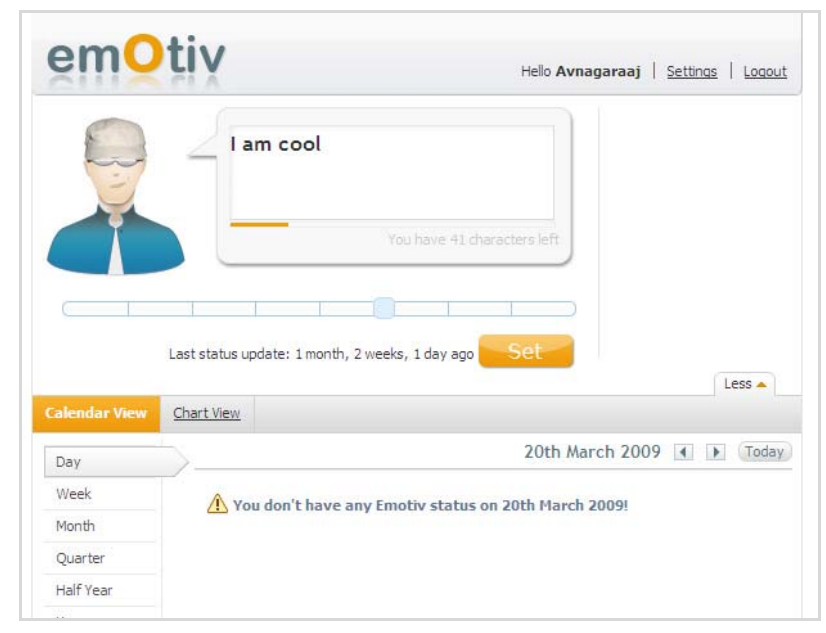

Figure 3: Emotiv - the initial face of Moodgroove.

There then evolved other versions of this model which split the mood measurement into an emotional scale and physical scale, so that people could track both parameters that contributed to their mood. More than a mood measurement, it was a measurement of the physical and mental stress levels of a user.

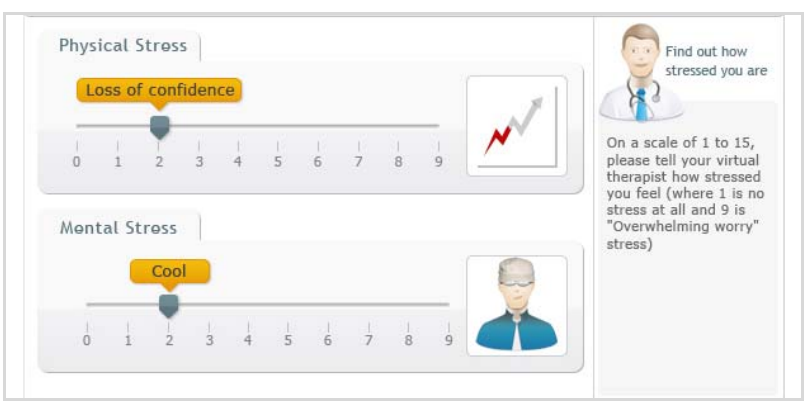

Figure 4: Emotiv - physical \& mental stress scales.

To improve the system's flexibility, we tried out a two-pronged approach of not only having a slider scale, but also having several words for a given scale value which users could choose from. This way, we ensured that the chances of finding a closer and more appropriate word to describe a feeling would be higher. A real time graph which would dynamically plot the user's feeling across time was also accommodated below the slider scale.

Another interesting feature addition at this point was the World Mood status. This automatically opened up the social side of the product - the inherent need to see how others around the world are feeling as compared to the user, and how many more people are feeling somewhat similar to the way he was - which brought in the comfort factor of being one among many. The UI design also evolved with each version to reflect the functionality. What started out with just a scale went on to adding a word option, a dynamic chart, and a world map.

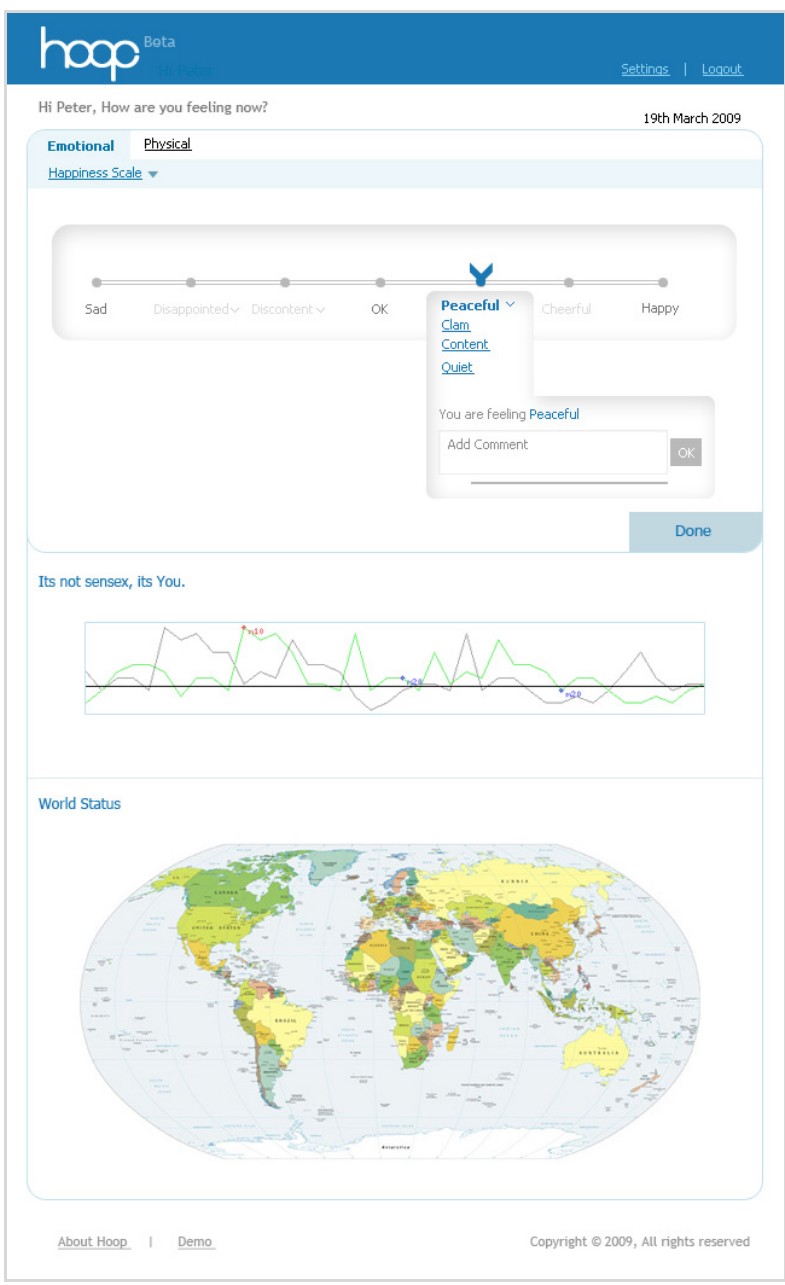

Figure 5: Hoop, initiation of two pronged approach.

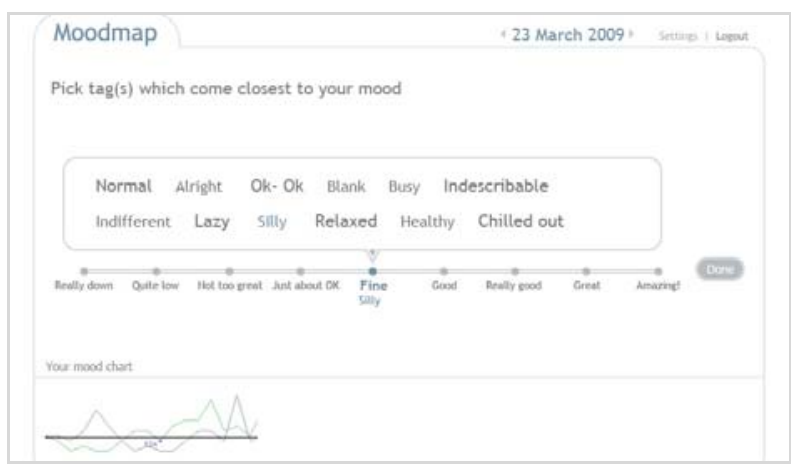

Figure 6: Moodmap

The product name too underwent its share of iterations. What started out as Emotiv, became Hoop, which further went on to Moodmap, and finally emerged as Moodgroove. The name Moodgroove suggests that most often we find ourselves stuck in a groove, unknowingly or knowingly, when it comes to our moods and feelings, and this is how the world sees us. This product helps you identify your groove which could thereby lead to self-realization or stimulate that much needed change in your life. 


\subsection{Moodgroove - Alpha version}

Having arrived at the name Moodgroove was a significant turning point in catapulting the product and user interaction to the next level. Not only did we build the main interaction for added flexibility, there was a lot of emphasis laid on the messaging and other aspects of the flow which would make it user friendly, right from the beginning.

The important additions in this version were the number of words to choose from. Here we established a hierarchy of parent and child tags. Parent tags were words which held the most popular mood, while child tags were other words which were close or similar to the parent tag.

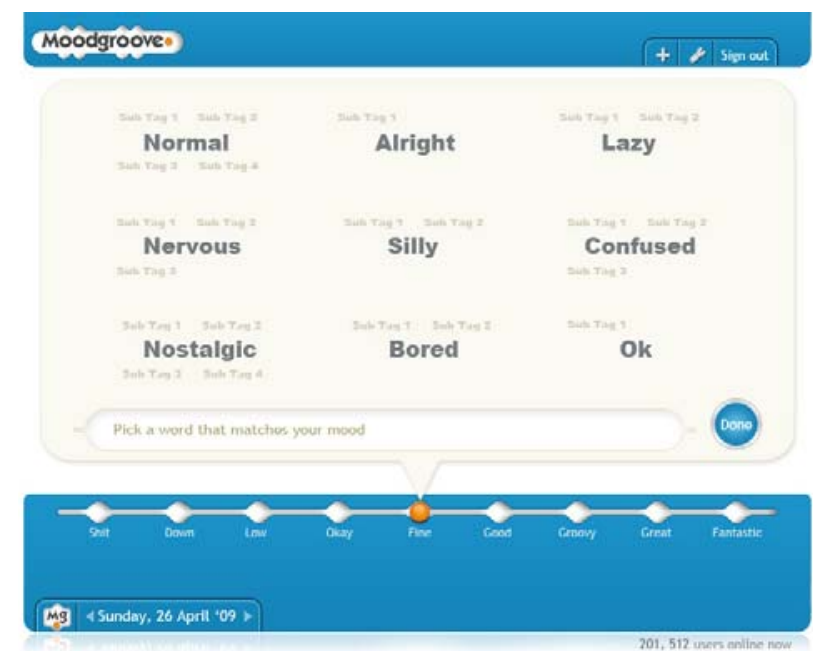

Figure 7: Moodgroove alpha version

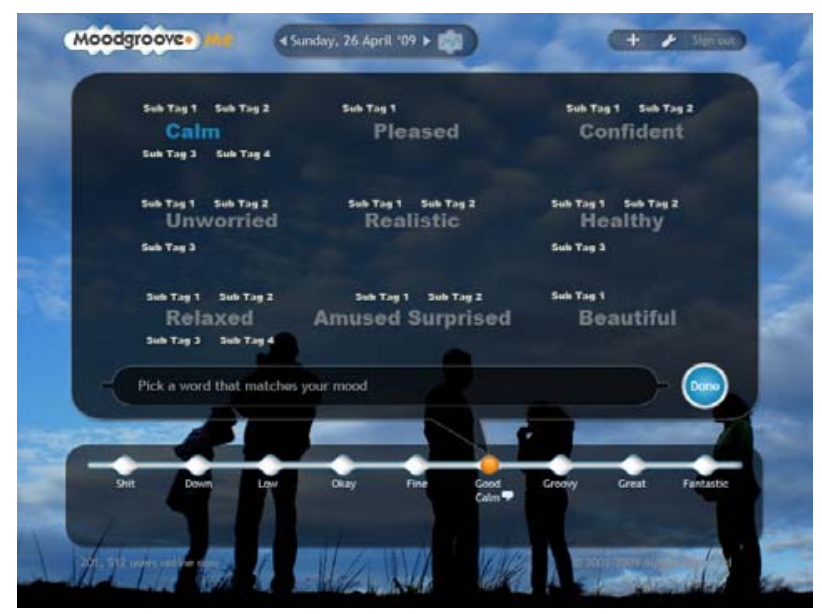

Figure 8: Alpha version with enriched UI.

Another functionality that got added at this stage was the search feature, which allowed users to search and locate the words they wanted. However, the assignment of words to a groove was fixed. So if a word didn't appear in the current groove, but it was found under another groove, the slider automatically jumped to reflect that groove selection.

Several variations of the blue and white model were tried out to see if we could make the UI look$\mathrm{n}$-feel cooler. Using images in the background suddenly lifted up the cool factor and made the experience more dramatic and closer to people and emotions.

\subsection{Moodgroove - Beta version}

A paradigm shift in the user experience and the concept of Moodgroove happened during the final phase of moving from the alpha to the beta version.

The introduction of a dynamic flash word cloud catapulted the system's experience factor to the next league - and users found it to be overwhelming. Having a dynamic word cloud also meant that there was no longer a limitation on the number of words the system could hold. The system saw a multi-fold leap in its vocabulary, and removing the hard pairing between words and grooves, brought in a lot more freedom for users to pair any word against any groove.

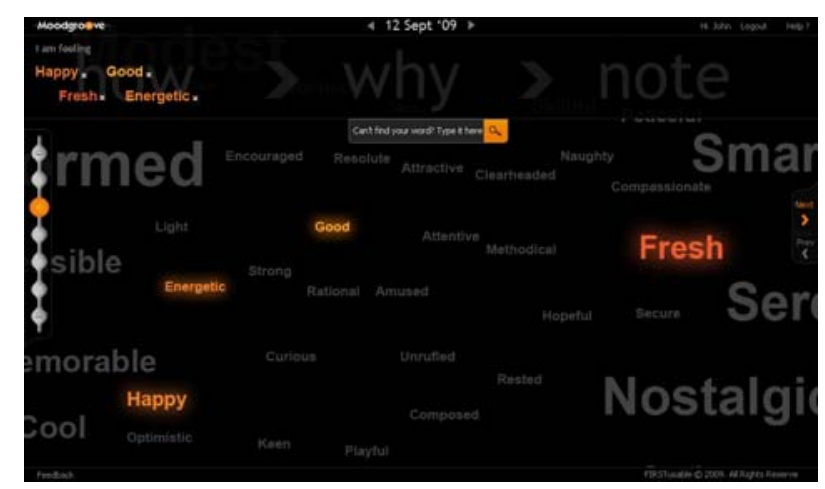

Figure 9: Moodgroove - with dynamic word cloud.

Another key factor added to the system was the addition of the second step which posed the next question - "Why are you feeling this way?". Users in beta version, can not only record their moods, but also associate reasons to them, which introduced more value to mood tracking. Moodgroove could now be used as a true introspection tool which could show patterns in your moods and their associations with reasons, an important medium to help in understanding mood swings [6].

Another critical improvement area for the beta version was the leap in visualization of reports. There was a need for not only showing how a user's moods had varied across a time period, but also provide more dimensions across which this mood data could be sliced. For e.g. how frequently a mood was felt, what were the most obvious reasons for a mood, etc - dimensions which made 
understanding mood data more intuitive, and aided in pattern finding and introspection.

At around this time, we also started experimenting with the idea of expressing mood in various other forms - art, music, haiku. These experiments are still underway, and we believe, being able to incorporate them effectively, can elevate the product to a whole new level.

\section{SYSTEM DESIGN}

\subsection{Core functionality}

Moodgroove in its current avatar sports a very minimalistic homepage with just a few pointers on what this product is about. It supports two types of login - through Moodgroove registered account, and through Facebook Connect.

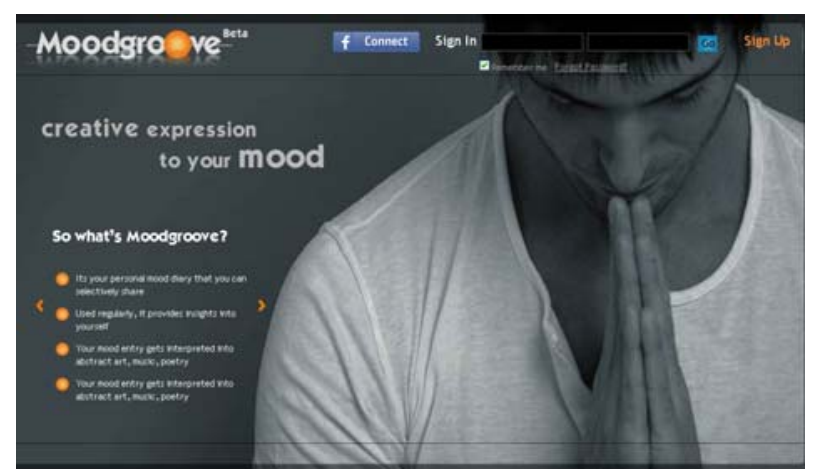

Figure 10: Home page - Moodgroove beta version.

On entering the system, user finds him/herself amidst a word cloud with the groove scale perched prominently on the screen - the starting point.

Moodgroove core functionality can be broken down into three steps -
1) "How are you feeling?" feeling this way?"
3) "Like to add something more?"

At the first step, the interaction involves a slider module free from any labels; but with emoticon-like partial faces on both extremes, to suggest the positive and negative mood portions on the scale. Users have to first pick a groove to indicate their overall mood. Each groove is associated with several word suggestions which appear as part of a word cloud in the space near the slider. One could pick from these, or search for a specific word in case he doesn't find it in the suggested word cloud.

The system supports search and suggest feature, which ensures that any word that a user searches for is either already present is the system or is taken as a user suggestion, so that the user doesn't have to bother about not finding his vocabulary.

Apart from the search/suggest feature, the system provides added flexibility when it comes to the number of words that can be picked by a user too. Moodgroove appreciates that users may not necessarily only have one kind of feeling at any moment - they could be going through a mix of emotions. To help them capture all these feelings irrespective of where they find themselves on the groove scale, the system allows users to pick up to 4 words to describe their feeling(s). They also have the option to remove or replace words, once picked.

Once users tell the system how they feel, they now proceed to the next step which is "Why are you feeling this way?" - the response to this is also in the form of words which users can pick from the word cloud. Alternatively, similar to the previous step, they can also search for specific words or suggest them to be added if they are not available in the system. The same word-limit is applicable to this step too, and so is the remove and replace functionality.

Moving on, users can now add an optional note to the mood entry. The note is primarily meant to be a short one or two liner (within 140 characters) which can also be posted to one's Facebook or Twitter account.

Having set all the above, the user proceeds to finally submit his mood entry. Editing entries in any step is allowed till this final submission happens.

\section{ENHANCEMENTS}

Post submission, Moodgroove shows the user his mood summary for the day - his mood entry along with the generated artwork, tune and poetry. These fall under the Art view of Mood Summary.

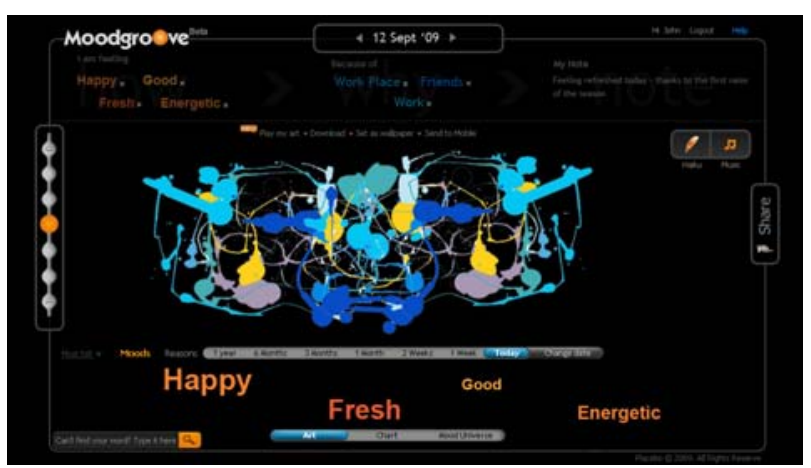

Figure 11: Sample Mood art. 
Although being a highly experimental area, there is an unspoken relationship between emotion and colour which has been previously explored by Jonathan Harris in We Feel Fine - assigning colours to the dots to indicate a range of positive and negative feelings. Taking the concept further, we tried to experiment with various colours and come up with a colour logic for each groove on the scale. A transformation of these colour shades using art generation algorithms into abstract art pieces for each mood entry is a unique contribution of this project in the space of cross-media moodmapping. A similar experimental technique was evolved to create a unique tune corresponding to a user's mood entry, with the help of the latest sound APIs available out there.

Users have the option to share any or all parts of this mood summary view on their social networking account or blog. Specifically, Mood entry (can be shared on Facebook, Twitter), Mood art (Facebook, Flickr, Twitpic, etc), Mood tune (Facebook, Myspace, any music sharing application, blog), Mood haiku (personal blog, Facebook, etc)

The other view is the Chart view which can be accessed using the slider at the bottom of the page. This chart plots mood variations for the given time frame. Seeing mood data across other dimensions has been enabled using multiple filters which slice moods and reasons across how frequently or infrequently they have been used in the given time frame.

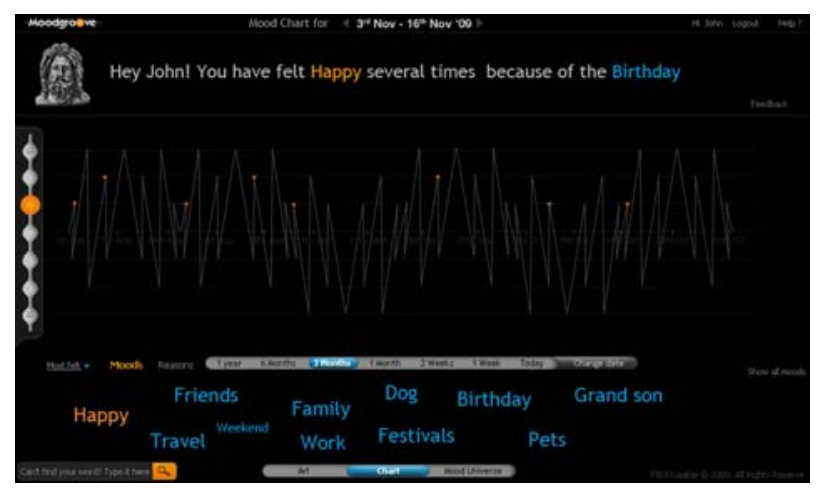

Figure 12: Chart view in Moodgroove

Art and Chart views pertain to user's own mood entries. The other view available is the Mood Universe, which displays a global view of moods

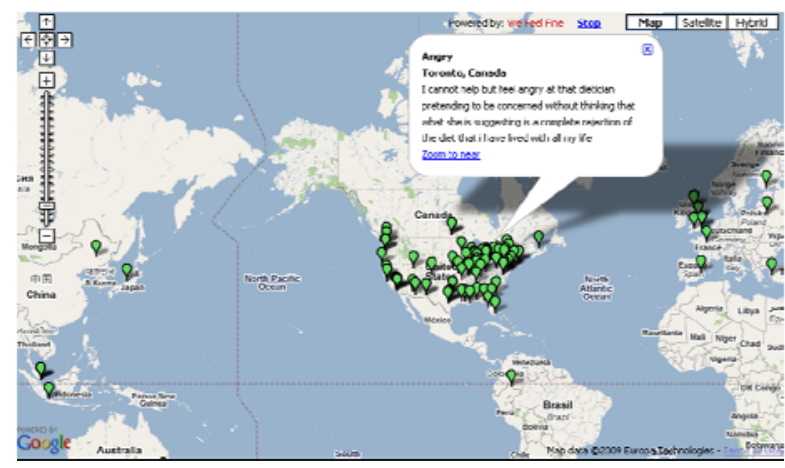

Figure 13: Mood Universe

across the world posted recently, on a Google Map interface - with markers pointing out the regions where they have been posted from. This mood display is dynamically updated in real time, and gives the user a larger perspective into the mood space - almost fosters a feeling of oneness with users across the world.

\section{CONCLUSION}

There is an inherent need in people to state, see and share opinions, thoughts, pictures, stories and their feelings through various mediums.

Moodgroove targets these perceived needs of users by extending a mood tracking application to a mood expression application. Our 18 months experience of designing Moodgroove has revealed that while dealing with sensitive topics like user feeling, it is essential to consider various aspects of human emotions. Our study described in this paper opens up several new possibilities of expressing mood through various manifestations. The domain of mood tracking is in a nascent stage and thus an interesting area to study.

\section{ACKNOWLEDGEMENT}

We would like to sincerely express our gratitude to Dr. Shyam Bhat (Psychiatrist), Jwala Narayanan (Psychologist), Ashritha Hattangadi (Special Educator), Dr. Narayan Reddy (Neurosurgeon), Peter Rockey (Technology Support) and Nagaraj Vijayarangan (Visual Designer) for their contribution to the conceptualization, design and development of Moodgroove. We sincerely thank all the users who helped us validate our concepts.

\section{REFERENCES}

[1] MoodViews: Tools for Blog Mood Analysis, http://moodviews.com/ 
[2] Sep Kamvar \& Jonathan Harris (2009) We Feel Fine - An almanac of human emotion,

[3] Parsons, J.; Ralph, P.; Gallagher, K. (July 2004), Using viewing time to infer user preference in recommender systems., AAAl Workshop in Semantic Web Personalization, San Jose, California .

[4] Larson, R., \& Csikszentmihalyi, M. (1983). The experience sampling method. New Directions for Methodology of Social and Behavioral Science, 15, 41-56.
[5] Suyog Deshpande, P. Yammiyavar "Mood Room - Intelligent Ambience" http://www.suyogdeshpande.com/moodroom.html

[6] Edery I: Circadian rhythms in a nutshell. Physiol Genomics 3:59-74, 2000. 